\title{
Automating Baseline Models for Code Compliance with Energy Conservation Building Code of India
}

\author{
Nikunj Shukla ${ }^{1}$, Mayank Bhatnagar ${ }^{1}$, Piyush Varma ${ }^{1}$, Hisham Ahmad ${ }^{1}$, Gurneet Singh Monga ${ }^{1}$, \\ Tanmay Tathagat ${ }^{1}$, Anurag Biswas ${ }^{1}$, Robin Jain ${ }^{1}$ \\ ${ }^{1}$ Environmental Design Solutions Private Limited, New Delhi, India
}

\begin{abstract}
Building codes are not always easy to interpret. This makes compliance documentation and evaluation a tedious and error prone process. In energy efficiency domain, some energy simulation tools facilitate compliance automation, but there are none for current Energy Conservation Building Code (ECBC). Since legislation mandates ECBC Compliance in several states in India, bridging this gap is essential.

This paper demonstrates Baseline Automation for ECBC (2017). The approach translates the Proposed Design Simulation Input Definition File (IDF) into a Baseline Case IDF using a Python based scripting language (Eppy). This automation serves as a productivity measure that frees up the energy modeler's time for pursuing useful design improvements.
\end{abstract}

\section{Introduction}

Building codes are developed and enforced to ensure safety and performance. Building codes, however, are not always easy to interpret. Hence demonstration of code compliance is often tricky, tedious and error prone process. In the absence of standard documentation processes, compliance evaluation becomes complex as well (Dimyadi \& Amor, 2013). Compliance automations can address this gap and substantially reduce time and effort. From the energy modeler's perspective, the automated routines minimize errors associated with manual inputs and standardize the documentation. From the regulator's perspective, the use of automated routines reduces effort associated with manually verifying code compliance against several rules.

The evolution of code compliance documentation has gone from checklists to simplified calculators to simulation-based reporting and more recently automated simulations.

Although, energy simulation approach ensures relatively robust outcomes, it is an input intensive, time consuming and technically demanding process. This process typically requires evaluating performance of a designed building against a benchmark building. Very often, modelling the benchmark building is a laborious process as it involves interpretation of the code and modifying the model accordingly. This only increases the energy modeler's effort.

To reduce this effort, there are few software and scripts in the domain that facilitate the compliance workflow. Some of these are for ASHRAE 90.1 Normative Appendix G Performance Rating Method (PRM) and Title 24 Compliance. For PRM, automated processes in DesignBuilder and OpenStudio ${ }^{\circledR}$ are popular. DesignBuilder has a built-in module that performs the automation process for PRM of ASHRAE 90.1 - 2007 and 2010. While most processes are automated, the module demands additional inputs for Baseline HVAC system. In OpenStudio®, measures (or scripts) must be loaded from the Building Component Library (BCL). The Baseline Automation measure requires use of standard space types built into OpenStudio ${ }^{\circledR}$ as templates for identifying spaces. In fact, research acknowledges that the measure to create ASHRAE 90.1-2013 Appendix G Baseline automation routine is one of the most complicated measures (Roth et al., 2018). CBECC-Com also uses OpenStudio ${ }^{\circledR}$ for automating Title 24 compliance. OpenStudio ${ }^{\circledR}$ has many independent measures in the BCL that may be used for compliance with specific requirements of Title 24 or PRM. The compliance automation tools discussed here use EnergyPlus simulation engine.

The Energy Conservation Building Code (ECBC) provides minimum requirements for energy efficient design of systems in commercial buildings in India. It was developed by the Government of India (GoI) in 2007. One of the compliance approaches is the Whole Building Performance (WBP) Approach. To document compliance using the WBP approach, an interactive web interface was developed. This application, ECOnirman WBP Tool, was developed under a collaborative program between the GoI and USAID (USAID ECO-III Team, 2011).

In 2017, the ECBC was updated. The updated ECBC is substantially different from the preceding edition. Since the ECOnirman WBP Tool is no longer under active development, it is not applicable to the updated code. Further in 2018, the ECBC Rules referencing the updated ECBC have been notified under the Energy Conservation Act (Ministry of Power, 2018). A report benchmarking performance of states in India, identifies 10 (out of 30) states that have notified ECBC. 9 of these 10 states are at an advanced stage of incorporating ECBC in municipal building bye laws. The notification implies mandatory compliance with ECBC to meet minimum energy performance criteria (AEEE, 2018).

In context of the legislation, documentation rigor and the unavailability of a compliance platform, the development 
of automation routine for updated ECBC and standardized reporting process is a first and essential step towards establishing a compliance ecosystem.

This paper demonstrates Baseline Automation for ECBC. The approach uses information from the Proposed Design model and automates the Baseline Case model compliant with WBP approach of ECBC.

As part of an ongoing activity for facilitating nationwide uptake of ECBC an online compliance portal has been developed. This portal serves as a platform for managing ECBC compliance process. The Baseline Case automation routine is a part of this development. While the ECBC does not mandate any simulation engine, the program requirements enforce the use of EnergyPlus as a simulation engine. As EnergyPlus is a robust and flexible tool (Crawley, Pedersen, Lawrie, \& Winkelmann, 2000) with many scripting frameworks, using it as the energy simulation engine has not been a constraint.

The program for online compliance portal has been discussed for context only and is not included in the paper's scope. Additionally, the paper focuses only on WBP compliance approach. Other alternative approaches and prescriptive compliance are outside the scope of this paper.

\section{Compliance with ECBC}

ECBC requirements are broadly organized into the following sections:
- Building Envelope

- Comfort Systems and Controls

- Lighting and Controls

- Electrical and Renewable Energy Systems

Requirements in each of these sections are further classified as Mandatory and Prescriptive requirements. In addition, a section on Whole Building Performance Method outlines modelling guidelines, HVAC system mapping, Chiller arrangements, lighting power adjustment factors and other rules.

ECBC compliance can be accomplished primarily in two ways; Prescriptive and WBP Approach. The WBP approach uses energy simulation to demonstrate compliance. To provide flexibility for compliance, some alternative compliance measures have been provisioned as well. Irrespective of the compliance path chosen, all Mandatory requirements must be met.

The WBP approach is similar to the PRM described in ASHRAE 90.1. The compliance is demonstrated through whole building simulation by comparing the performance of Baseline Case model with that of the Proposed Design model. The compliance procedure requires modeling a Baseline Case consistent with the Prescriptive requirements while the Proposed Design model reflects actual design specifications.

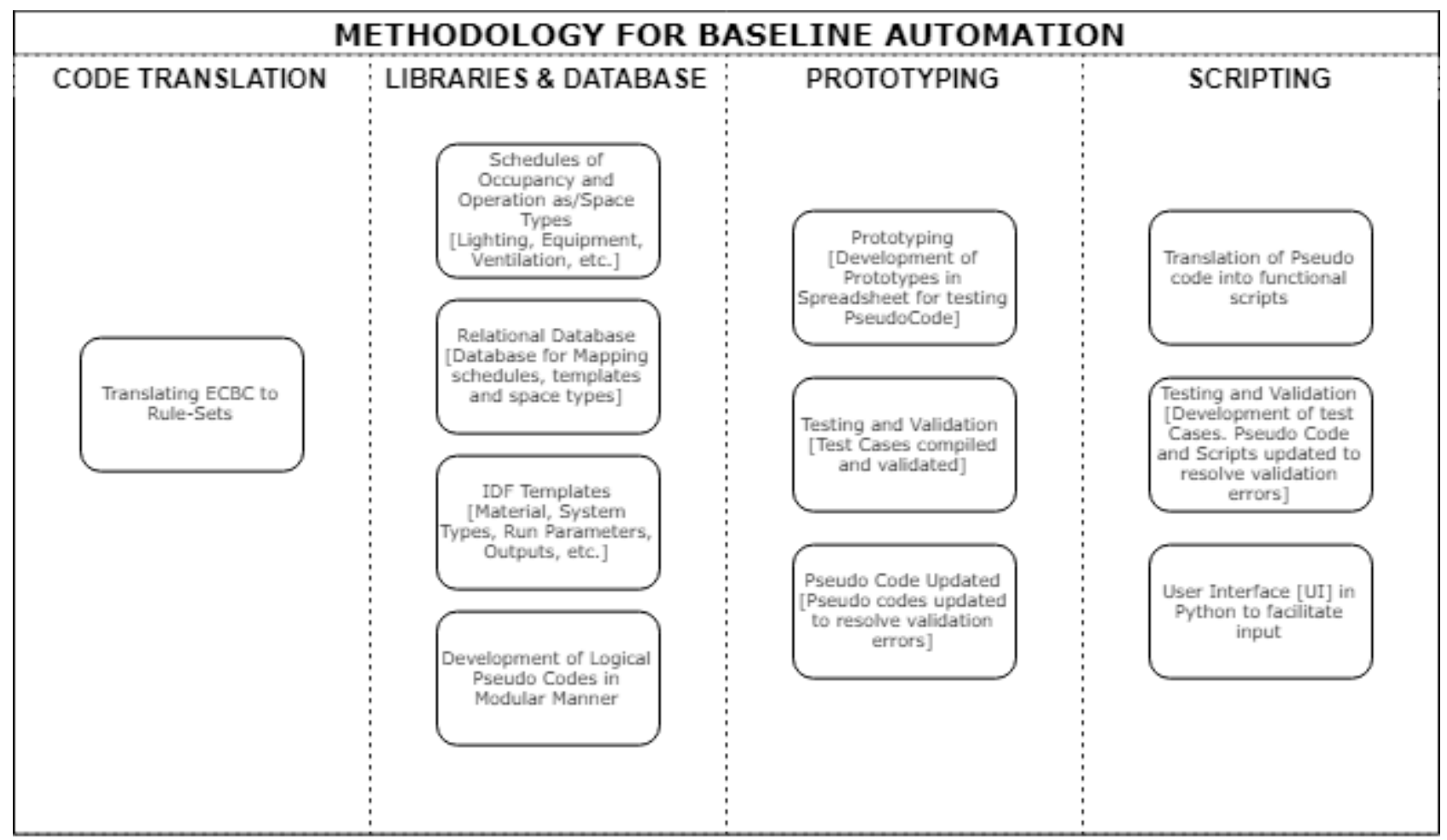

Figure 1: Implementation methodology for developing the Baseline Case Automation Routines. Methodology is spread across 4 major activities: Code translation, Preparation of database and libraries, Prototyping, and Scripting. 


\section{Methodology}

ECBC has been interpreted as rule-sets. These rule-sets are accompanied with database tables. The database serves as lookup tables while the rulesets define the logic for lookup and other actions. These rule-sets are based on key project information such as location (climate and latitude), above grade area, building typology, occupancy, conditioned status, compliance level, etcetera. These factors serve as primary filters that define baseline construction assemblies, HVAC system types, equipment efficiencies, etc. Another key building component is the space type. The ECBC includes a list of common space types indicative of space functions (say conference room). To complement this database, IDF templates of schedules (occupancy and operation), lighting, equipment, infiltration, system types, etcetera have been created as well. Additionally, IDF templates for Construction assemblies and Baseline Systems have been created in EnergyPlus. All this information has been compiled as a relational database.

Some of these automation logics are simple calculators and lookup actions. These simple calculators have been implemented as a spreadsheet prototype for logical testing before implementing into the code. The outcomes from the spreadsheet prototype have been manually validated. The spreadsheet prototype is quick to implement, easier to deploy and facilitates testing. The prototype reduced coding effort and associated debugging time. The prototyping effort complemented the pseudo code compilation. The prototype and pseudo code have been compiled module by module, i.e. in an incremental manner.

The rule-sets, prototype and templates set the foundation for programming logic scripted in Eppy. Eppy leverages Python, a popular 'readable' programming language that facilitates scripting tasks. The Eppy package facilitates accessing and modifying EnergyPlus objects, significantly reducing and simplifying coding effort (Roth et al., 2018). The Baseline Automation program contains several modules fulfilling smaller tasks. The intention for a modular approach is to facilitate testing and de-bugging. Test cases have been developed and tested for validation. Several test trials were conducted for validation. These tests compared the automated Baseline Case IDF with manually derived outcomes from the ECBC. The distinct phases of implementation have been outlined in Figure 1. A simplified User Interface (UI) (Figure 2) in Python has been developed for facilitating input. The compliance output is reported on the online compliance portal.

\section{Process Workflow}

The Baseline Automation process requires a user input of the Proposed Case IDF. Before initiating the Proposed Design model, the user is provided with naming convention and Zone List assigning procedure. Zone List is an object in IDF which represents a space typology to which different spaces can be assigned (LBNL, 2009). Figure 3 illustrates a group of zones attached to a Zone List (Example: Obj1 or Co-Lobby-Day). Name field identifies an object in ZoneList (which is assigned as per naming convention) and the Zone Name fields indicate the zones assigned to this Zone List. This user workflow of assigning zone to zone lists is similar to how OpenStudio® uses Space Types and Space Name. This Zone List is further mapped with Schedules and other zone-specific information such as Lighting, Equipment, schedules, etc. from the database tables. The naming convention is critical as it allows the program to read zones and assign zone related information.

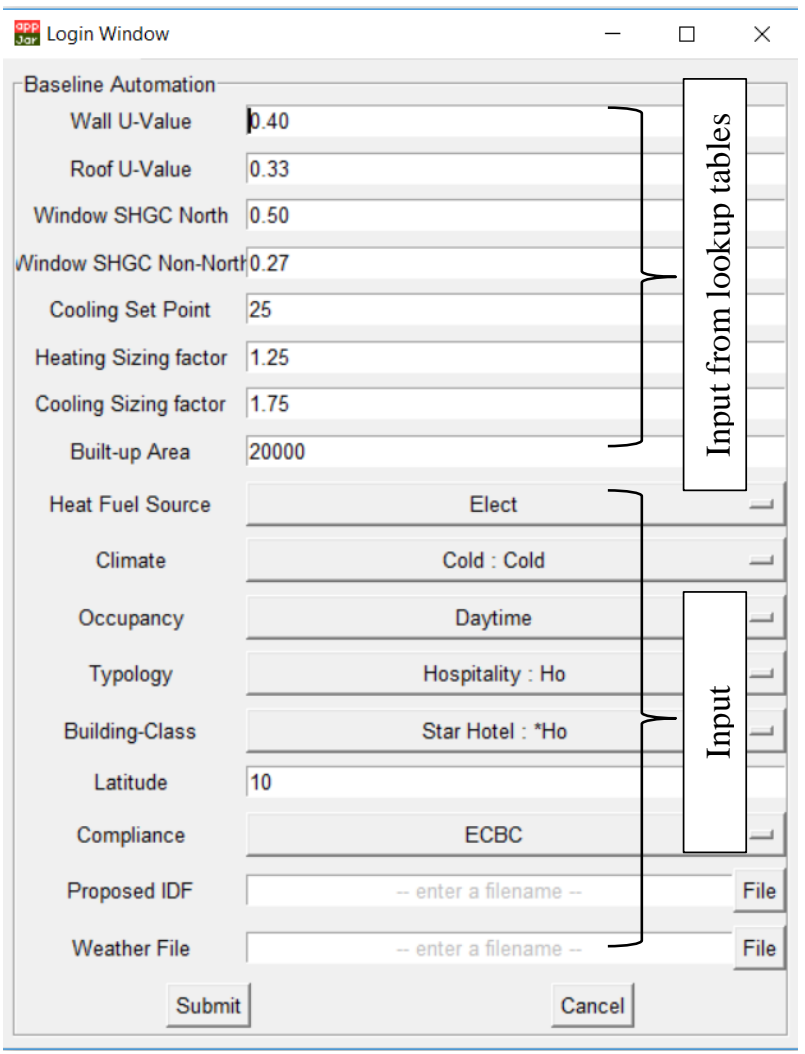

Figure 2: User Interface (UI) developed in Python.

\begin{tabular}{|c|c|c|c|c|}
\hline \multicolumn{5}{|c|}{ Explanation of Object and Current Field } \\
\hline \multicolumn{5}{|c|}{$\begin{array}{l}\text { Object Description: Defines a list of thermal zones which can be referenced as a group. The Zonel } \\
\text { may be used elsewhere in the input to apply a parameter to all zones in the list. } \\
\text { ZoneLists can be used effectively with the following objects: People, Lights, } \\
\text { ElectricEquipment, GasEquipment, HotwaterEquipment, Zonelnfiltration:DesignFlowRate, } \\
\text { ZoneVentilation:DesignFlowRate, Sizing:Zone, ZoneControl: Thermostat, and others. }\end{array}$} \\
\hline \multicolumn{5}{|c|}{ Field Description: Name of the Zone List } \\
\hline Field & Units & Obj1 & Obi2 & Obj3 \\
\hline Name & & Co-_obby-Day & Bu-0ff $(<10 \mathrm{~K})$. & Co-Stairway-Day \\
\hline Zone 1 Name & & Bagsement_Lobby & FF_Cabins - & Basement_Lift \\
\hline Zone 2 Name & & FF_Lobby & FF_WS1 & Basement_Stairca \\
\hline Zone 3 Name & & F_Lobby & FF_WS2 & FF_Lift \\
\hline Zone 4 Name & & Stilt_Atrium & SF_Cabins & FF_Staircase \\
\hline Zone 5 Name & & Still_Lobby & SF_WS1 & SF_Lift \\
\hline Zone 6 Name & & Stilt_Reception & SF_WS2 & SF_Staircase \\
\hline Zone 7 Name & & Stilt_Visitors Gallery & TF_DR1 & Stilt_Lift \\
\hline Zone 8 Name & & TF_Corridor & TF_DR2 & Stilt_Staircase \\
\hline Zone 9 Name & & TF_Lobby & TF_DR3 & TF_Lift \\
\hline Zone 10 Name & & Terrace_Lobby & TF_PA & TF_Staircase \\
\hline Zone 11 Name & & & & Terrace_Lift \\
\hline Zone 12 Name & \\
\hline \multicolumn{3}{|c|}{$\begin{array}{c}\text { Space Type } \\
\text { (as/convention) }\end{array}$} & \multicolumn{2}{|c|}{$\begin{array}{l}\text { Spaces assigned to } \\
\text { the Space Type }\end{array}$} \\
\hline
\end{tabular}

Figure 3: Modelling convention that uses pre-defined names for Zone-Lists (or Space Types) and maps Zones

(or Spaces) as a List. This is similar to Space Type object implementation in OpenStudio ${ }^{\circledR}$. 
The Proposed Case IDF created as per Zone List rules is provided as input to the tool. Other inputs like Building typology, Daytime/24-hour occupancy, Location, etc. are provided as well through a user interface (Figure 2). Before proceeding, the script runs a validation check for compliance with mandatory requirements and zone-list acceptance. In case any of these checks are not met, the script returns an error. A valid Proposed Case IDF must be provided to proceed further. After the validations are met, the tool makes copies of the Proposed Case IDF. On both these files the Simulation Environment object and default schedule objects are applied. One of these files is saved as Modified Proposed Case IDF, while the other is saved as Baseline Case IDF (See 1 in Figure 4). The Baseline Case IDF then undergoes modification for setting Baseline Construction Assemblies, Lighting Power and Controls, and mapping HVAC System types. At this stage the HVAC systems in the Baseline Case IDF are set to autosize. Since the code mandates System Type efficiencies as per capacity, a sizing run is performed. The outcome of sizing run informs the HVAC System capacities. The HVAC System capacities and efficiencies are updated by program based on code stipulations. The updates to equipment efficiency and system types are saved as Modified Baseline Case IDF (See 2 in Figure 4). The Modified Baseline Case IDF and the Modified Proposed Case IDF are simulated on the EnergyPlus simulation engine. The outcomes of the Modified Baseline Case IDF and the Modified Proposed Design
Case IDF are evaluated as a ratio of Energy Use Intensity (EUI). This ratio defines the compliance level achieved.

\section{Unique challenges}

The WBP Method is inspired by PRM. Although scripting frameworks and/or tools for PRM and Title 24 exist, automating the Baseline for ECBC has been a challenge due to several factors. Some of these challenges outline the novelty in this approach.

The Baseline Automation routine is a part of an online compliance framework. Due to various reasons the online portal does not allow a user interface for building geometry input yet. Since the modeling environment is not available, the framework accepts the Proposed Design model created by the user as IDF. The compliance portal uses scripting routines to process this into a Baseline Case IDF. Therefore, the modeling environment and the energy simulation engine are essentially de-coupled. This is unlike other automations where both the script/plugin and the modeling environment reside in one system. The challenge in a de-coupled system is constraining the user input into a script-readable format. To overcome this challenge, a naming convention addressing the space types referenced in the standard has been compiled. Process workflows for creating ZoneList objects have been detailed for EnergyPlus interfaces in DesignBuilder, OpenStudio and native IDF Editor UI. This process has been explained in the Process Workflow section.

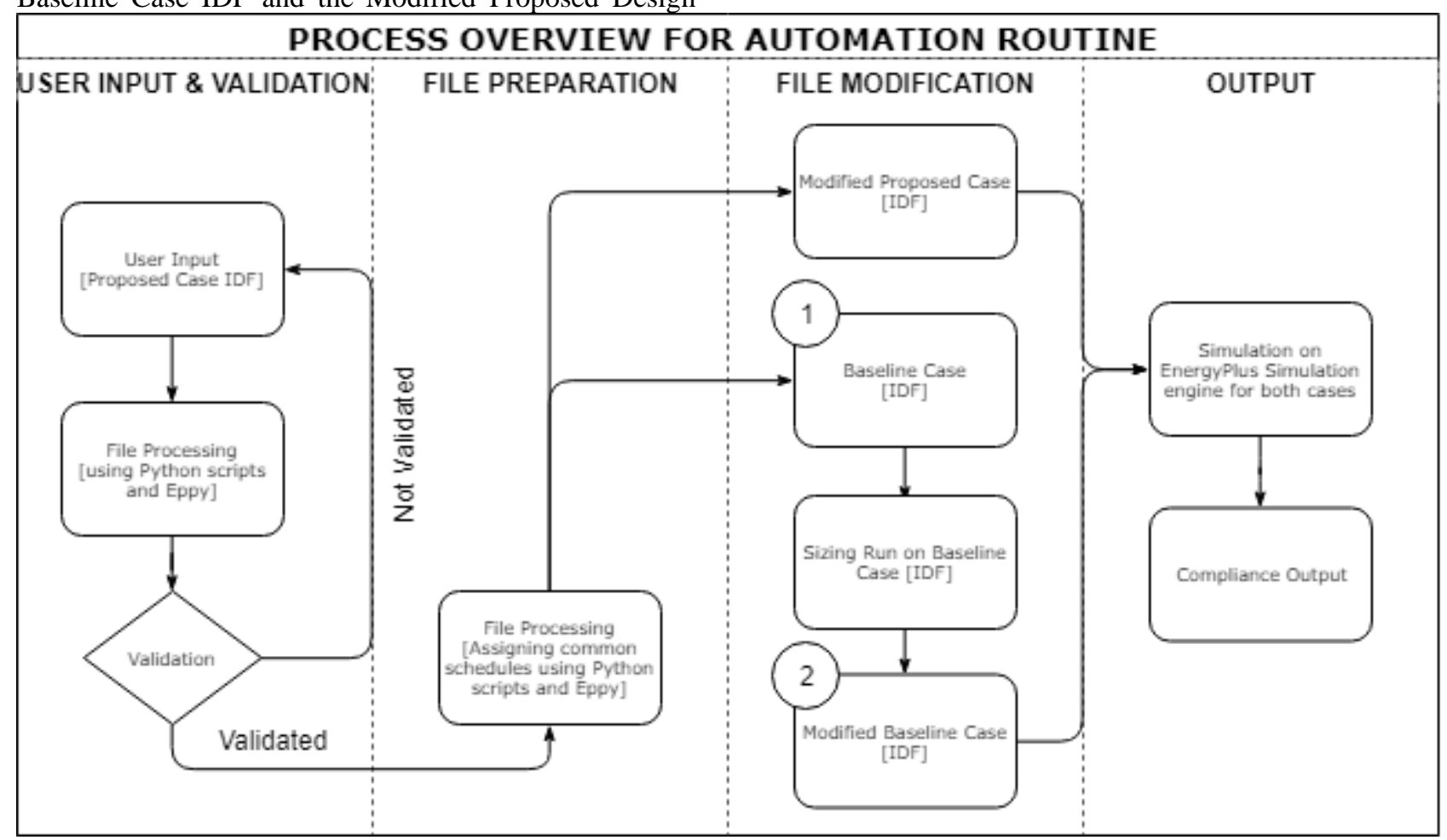

Figure 4: The flowchart gives an overview of the automation process. The input file is validated and processed for modification. The modified files are simulated for generating output. 
The ECBC was envisaged as an inclusive code. It attempts to address diversity in political, jurisdictional and socio-economic aspects of the commercial building sector in India. One unique challenge in addressing the jurisdictional context has been the Baseline Case Chiller Selection Ruleset. The ECBC mandates Water-cooled Chillers above a threshold capacity. However, there are some jurisdictions that face severe water crisis, and Water-Cooled Chillers are not technically feasible. To include such jurisdictions in the purview of the code, exceptions for using Air-cooled chillers have been outlined. Similarly, there have been provisions to include high performance single glazed units for unconditioned/naturally ventilated buildings that are essentially low cost. Accounting for several such exceptions requires complex rule sets. The compiled rulesets account for many such exceptions and ensure the intent of the code.

\section{Conclusions and Way Forward}

The approach demonstrates that the automation routine successfully builds a Baseline Case model from a Proposed Design model. The automation routine is helpful in standardizing and streamlining compliance workflows. The approach saves energy modeler's time and effort as well.

The ECBC has several exceptions tailored for the Indian context, as it is an inclusive code by design. and it has many instances of exceptions in the code. Addressing several exceptions makes the case for standardizing compliance processes and automation routines even stronger.

This approach can be applied to Proposed Design Model built in IDF Editor (EnergyPlus), DesignBuilder and OpenStudio®. Other interfaces have not been tested for this routine. Further, other simulation engines such as DOE-2 have not been included yet. Other simulation interfaces and engines may be taken up for future upgrades.

The Performance Rating Method - Reference Manual documents the rule sets and code interpretations for the reference of Building Simulation community (Goel, Rosenberg, Liu, Contoyannis, \& Czech, 2016). There is potential to develop a similar document for the benefit of energy modelers and developers associated with ECBC compliance processes. This is expected to facilitate the integration of rulesets into popular software. There is potential to integrate existing PRM automations in the workflow to facilitate single models for ECBC and PRM compliance as well.
It is anticipated that different states (or jurisdictions) in India will adopt the updated ECBC with contextual changes. There will be potential to integrate these customizations as scripts in the future.

\section{Acknowledgement}

The development of the Baseline Automation Routine for ECBC is part of a larger compliance framework commissioned by the United Nations Development Programme (UNDP) in India on behalf of the Bureau of Energy Efficiency (BEE).

\section{References}

AEEE. (2018). State Energy Efficiency Preparedness Index. New Delhi.

Crawley, D. B., Pedersen, C. O., Lawrie, L. K., \& Winkelmann, F. C. (2000). EnergyPlus: Energy Simulation Program. ASHRAE Journal, 50-56.

Dimyadi, J., \& Amor, R. (2013). Automated Building Code Compliance Checking - Where is it at? Proceedings of CIB WBC 2013, (May 2014), 172185. https://doi.org/10.13140/2.1.4920.4161

Goel, S., Rosenberg, M., Liu, B., Contoyannis, D., \& Czech, N. (2016). Developing a Detailed Ruleset for Use in Automating the Performance Rating Method of ASHRAE Standard 90.1-2010. In SimBuild 2016 (pp. 205-213). Salt Lake City: ASHRAE and IBPSA-USA.

LBNL. (2009). Input Output Reference (Build 4010). U.S. Department of Energy. Retrieved from https://energyplus.net/sites/all/modules/custom/nre 1_custom/pdfs/pdfs_v8.9.0/InputOutputReference. pdf

Ministry of Power. ECBC Rules 2018, Pub. L. No. D. L.33004/99, 181 (2018). India. Retrieved from https://beeindia.gov.in/sites/default/files/ECBC Rules_Gazette Copy.pdf

Roth, A., Bull, J., Criswell, S., Ellis, P., Glazer, J., Goldwasser, D., ... Reddy, D. (2018). Scripting Frameworks For Enhancing EnergyPlus Modeling Productivity. In SimBuild 2018 (pp. 312-319). Chicago.

USAID ECO-III Team. (2011). ECOnirman Whole Building Performance User Manual. USAID ECOIII Program. New Delhi. Retrieved from http://eetools.in/ECOnirman_WBP_UserManual.p df 